\title{
Prevalence of Helicobacter pylori Antibodies in Egyptians with Idiopathic Thrombocytopenic purpura and in the General Egyptian Population: A Comparative Study
}

\author{
Nesren F. Hanafi ${ }^{1}$, Irene L. Mikhael ${ }^{2}$ and Doreen N. Younan ${ }^{3 *}$ \\ ${ }^{1}$ Department of Microbiology, Faculty of Medicine, Alexandria University, Alexandria, Egypt \\ ${ }^{2}$ Department of Haematology, Medical Research Institute, Alexandria University, \\ Alexandria, Egypt \\ ${ }^{3}$ Department of Clinical Pathology, Faculty of Medicine, Alexandria University, \\ Alexandria, Egypt \\ *Corresponding author email id:
}

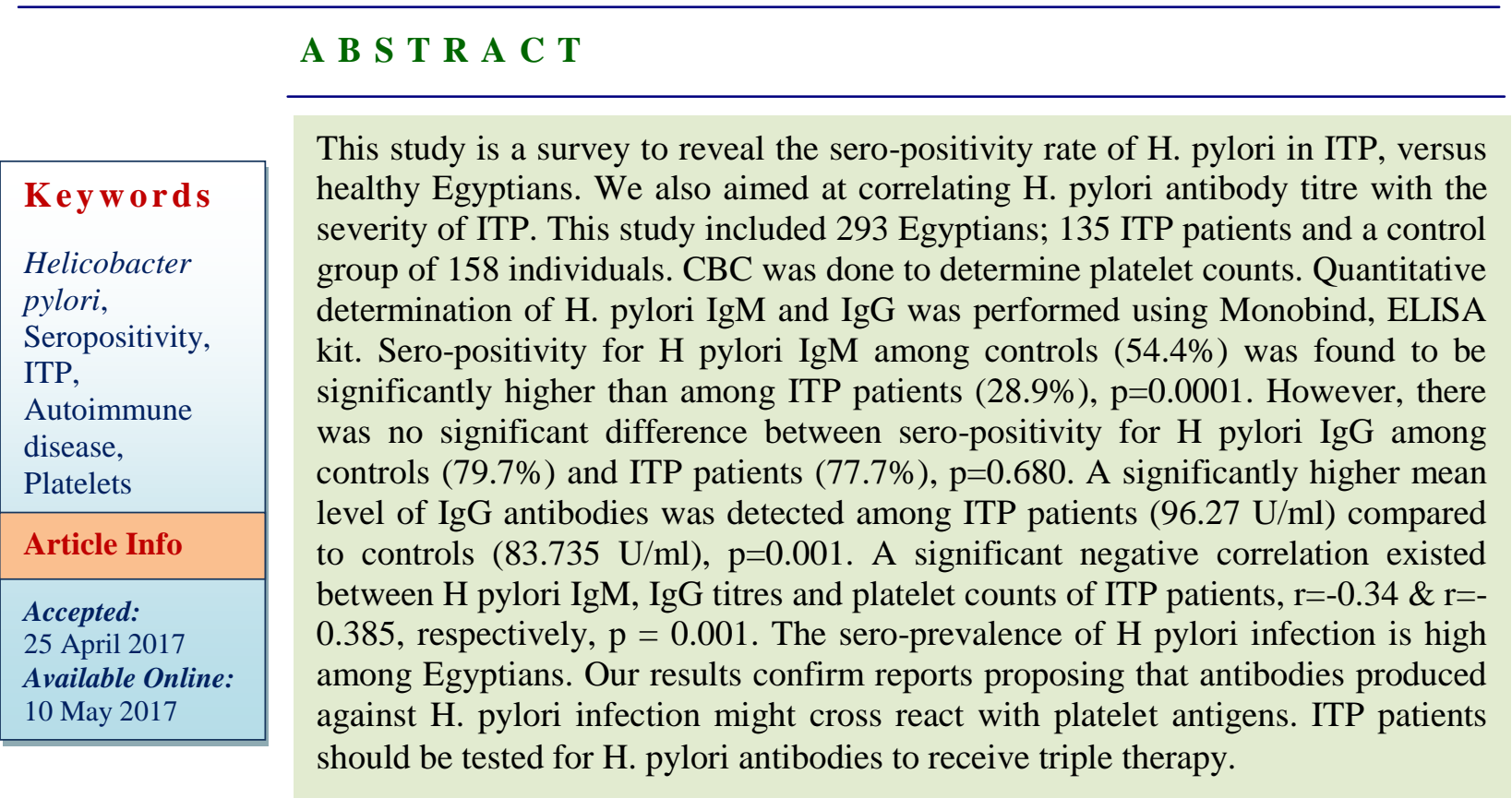

\section{Introduction}

Immune thrombocytopenic purpura (ITP) is an autoimmune bleeding disorder resulting from antibodies against platelet surface glycoproteins, resulting in their destruction. Several microbial agents causing chronic infections, such as human immunodeficiency virus (HIV), hepatitis $\mathrm{C}$ virus (HCV) and helicobacter pylori ( $\mathrm{H}$ pylori) have been shown to be associated with ITP (Hasni, 2012).

Previous studies suggest that infectious agents may influence the occurrence or the course of some autoimmune diseases (Rizzo et al., 2014). There are several proposed mechanisms by which microbial organisms 
can lead to loss of self-tolerance; such as molecular-mimicry, when shared amino acid sequences between microbial antigens and host proteins lead to generalized triggering of immune response against both the host proteins and microbial antigens (Cooke et al., 2008). Other proposed mechanisms leading to triggering of autoimmunity include polyclonal activation, epitope spread, bystander activation and super-antigens (Amital et al., 2008).

Helicobacter pylori are widely prevalent, spiral Gram negative bacteria which were discovered, as human pathogens, by Marshall and Warren in 1982 [Marshall and Warren, 1984]. Studies have indicated that $H$ pylori typically infect the gastric mucosa, and so their presence is associated with a variety of gastrointestinal diseases including gastritis, duodenal and gastric ulcers, non-ulcer dyspepsia, and gastric adenocarcinoma and lymphoma (Zhong et al., 2016).

Helicobacter pylori infection has a high prevalence globally, ranging from 50 to $80 \%$. Usually acquired early in life, it is characterized by long incubation period. Most of the infected cases remain asymptomatic for decades. Clinically presented cases are commonly associated with gastritis and peptic ulcer disease (Kao et al., 2016).

Infected hosts' immune response, not only fails to resolve the infection, but may contribute to the severity of the disease. This pathogenicity involves stimulation of $\mathrm{T}$ helper one induced inflammation. Some studies document that $\mathrm{H}$ pylori infection down regulates the host's immune response and also some researchers suggest the contribution of $\mathrm{H}$ pylori to some autoimmune disease' development (Hasni et al., 2011). Multiple publications have attributed a role for $H$. pylori infection in causing a variety of extraintestinal manifestations (Sherman et al.,
2005), (Wong et al., 2014), (Bruscky et al., 2014).

Effective diagnostic modalities and treatment strategies are currently available and have proven to be effective in detecting and eradicating of $\mathrm{H}$ pylori infections. Organism removal by antimicrobial therapy is correlated with the resolution of symptoms and cure of the disease. However, traditional treatments of ITP involve the use of immunosuppressive agents and immunoglobulin therapy (Shmuely et al., 2016).

Inspite of the conflicting data, some researchers reported high association of $\mathrm{H}$ pylori infection prevalence with many autoimmune diseases such as ITP, atrophic gastritis and mucosa associated lymphoid tissue (MALT) lymphoma. Autoimmune diseases' unclear etiology has been justified by the hypothesis of being induced due to exposure to viral, bacterial or chemical agents, in a genetically predisposed individual (Hasni, 2012).

The present study aims at reporting the prevalence of $\mathrm{H}$ pylori antibodies (IgM and IgG) in the general Egyptian population and in ITP patients and, also, determines the correlation between quantitative estimation of their tire in sera of infected ITP patients and severity of thrombocytopenia.

\section{Materials and Methods}

\section{Study design}

Cross-sectional observational study.

\section{Subjects}

The present work was conducted in Alexandria University Teaching Hospital and Medical Research Institute, in Northern Egypt. This study received ethical approval 
from the Institutional Review Board at Faculty of Medicine, Alexandria University in Egypt and written informed consents were obtained from all participants before enrollment in the study. The identification information of all subjects was kept confidential and was protected from the public. This work has been carried out in accordance with the Code of Ethics of the World Medical Association (Declaration of Helsinki) for experiments involving humans.

One hundred and thirty five adult ITP patients (above 18 years of age), presented to the Haematology Clinic at Medical Research Institute of Alexandria, were recruited in this case-control study. They comprised 90 females and 45 males (male to female ratio of $0.5: 1)$, their ages ranged between 18 and 56 years.

Immune thrombocytopenic purpura was diagnosed on the basis of the presence of isolated thrombocytopenia $(<100 \times 109 / \mathrm{L})$ with or without megakaryocytic hyperplasia in the bone marrow. Other causes of thrombocytopenia (drugs, pseudothrombocytopenia, HBV, HCV, HIV, malignancy and collagenic diseases) were all excluded.

One hundred and fifty eight age and sex matched Egyptians served as a control group. They comprised 84 females and 74 males with a male to female ratio of $0.9: 1$, their ages ranged between 18 and 57 years. They were all non-thrombocytopenic, apparently healthy without dyspeptic complaints.

None of the included subjects had previously received antibiotics (commonly used in antiH. pylori therapy), $\mathrm{H} 2$ blockers or proton pump inhibitors (PPIs) in the three months preceding this study. Patients with history of gastric resection/ vagotomy, those with complicated peptic ulcer disease and those considered at bleeding risk, were also excluded.

\section{Laboratory tests (Hasni, 2012)}

Quantitative determination of $\mathrm{H}$ pylori specific antibodies of the $\operatorname{IgM}$ and $\operatorname{IgG}$ types, in sera of both ITP patients and controls, was done in Alexandria University Teaching Hospital, using commercial enzyme immunoassay kits (Accu Bind ELISA micro wells, product codes; 1525-300 IgM and 1425-300 IgG, Monobind Inc, Lake Forest, CA 92630, USA). The reagents were stored unopened at 40C. Repeated freezing and thawing of sera was avoided. Icteric and turbid samples were not used (manufacturer's precautionary advice). A reference curve was drawn on a linear graph paper; using 5 anti $\mathrm{H}$ pylori calibrators supplied within the kit, to determine the concentration of $\mathrm{H}$ pylori $\mathrm{IgM}$ and $\mathrm{IgG}$ in unknown specimens (Hasni et al., 2011).

The presence of IgG antibodies to $H$. pylori was documented when the serum level exceeds $20 \mathrm{U} / \mathrm{ml}$ while the presence of IgM antibodies to $\mathrm{H}$ pylori was documented when the serum level exceeds $40 \mathrm{U} / \mathrm{ml}$ (according to manufacturer's recommendations). Specimens with concentrations greater than $100 \mathrm{U} / \mathrm{ml}$ were additionally diluted $1: 5$ or 1:10 with the supplied serum diluent and the final result was obtained after multiplication by the dilution factor. A positive result does not indicate gastrointestinal disease and does not distinguish between colonization and infection. Similarly, a negative result does not eliminate the absence of $\mathrm{H}$ pylori infection. A low titre of antibody may be related to early stages of colonization.

Complete blood counts (CBCs) were done by Sysmex ${ }^{\circledR}$ STKS (Coulter Corporation Miami, Florida, USA). Bone marrow aspiration was done to selected ITP patients. 
Although the presence of $\mathrm{H}$ pylori bacilli in gastric biopsies is the gold standard of $\mathrm{H}$ pylori detection, we preferred blood antibody detection due to following reasons; endoscopy might cause unexpected bleeding in thrombocytopenic patients, especially in those whose platelet counts are less than $50 \times 109 / \mathrm{L}$, and urea breath test could not allow the detection of $\mathrm{H}$ pylori infection retrospectively.

\section{Statistical Analyses (Binu et al., 2014)}

Data were collected, tabulated and statistically analyzed using SPSS 20.0 (SPSS Inc., Chicago, IL, USA) and Microsoft Office Excel 2010 (Microsoft Cor., Redmond, WA, USA). Continuous data are expressed as mean $\pm \mathrm{SD}$ and median (range). Categorical data are expressed as a number (percentage). Continuous variables were checked for normality by Kolmogorov-Smirnov test. Mann-Whitney U test was used to compare two groups of non-normally distributed data and independent $\mathrm{t}$ test for parametric data. Percent of categorical variables were compared using Chi-square $\left(\chi^{2}\right)$ test and Fischer exact test for $>50 \%$ of cell count $<5$. Comparing studied groups, one way ANOVA test was used for parametric data and Kruskal Wallis test for non-parametric data. Spearman correlation was done for non-parametric correlation between Ig titres and platelet counts. All tests were two tailed. $p \leq 0.05$ was considered statistically significant, $\mathrm{p}<0.01$ was considered highly statistically significant and $p>0.05$ was considered non-statistically significant.

\section{Results and Discussion}

The present study comprised 135 ITP patients; 45 males (33.3\%) and 90 females $(66.7 \%)$ with a mean age \pm SD of 30.3 years \pm 9.6. A control group of 158 healthy volunteers; 74 males (46.8\%) and 84 females
(53.2\%) have been included matched for age $(p=0.698)$ and sex $(p=0.101)$. The platelet counts of ITP patients ranged between 3,000 and $104,000 / \mathrm{cmm}$ with a mean $\pm \mathrm{SD}$ of $29,333 / \mathrm{cmm} \pm 23,662$, while those of healthy controls ranged between 159,000 and $560,000 / \mathrm{cmm}$ with a mean $\pm \mathrm{SD}$ of $354,300 / \mathrm{cmm} \pm 30,501$.

The prevalence of seropositivity for antihelicobacter IgM among healthy controls was $54.4 \%$ (86/ 158), while it was lower among ITP patients; $28.9 \%$ (39/ 135), without a statistical significant difference between the 2 groups $(p=0.291)$. On the other hand, the prevalence of anti-helicobacter IgG was lower among ITP patients; $77.8 \%(105 / 135)$ compared with controls; $79.8 \%$ (126/ 158), still with no statistical significant difference revealed $(\mathrm{p}=0.680)$, as shown in table 1 .

The prevalence of anti-helicobacter IgM among all sero-positive ITP patients was found to be $36.1 \%$, while that among seropositive controls was $60.6 \%, \mathrm{p}=0.004^{*}$. For anti-helicobacter $\mathrm{IgG}$, it was found to be 97.2\% among all sero-positive ITP patients and $88.7 \%$ among sero-positive controls, $\mathrm{p}=0.54$. $($ Table 1$)$

Furthermore, platelet counts of $H$. pylori seropositive ITP patients was found to be significantly lower than that of $H$. pylori seronegative ITP patients $(\mathrm{p}<0.05)$ (Table 2$)$.

Intending to study the effect of chronic or past $\mathrm{H}$ pylori infection, we excluded IgM positive cases in both groups (as presence of IgM denotes acute infection). The comparison revealed a statistical significant increase in ITP cases; $51.1 \%$ (69 out of 135) versus $35.4 \%$ among controls (56 out of 158) who were concomitantly $\operatorname{IgG}$ positive and IgM negative (denoting past or chronic infection) (X2 $=22.3, \mathrm{p}=0.0001)$. While, IgM only positive cases (denoting a recent or acute 
infection, evidenced by absence of concomitant IgG positivity), were found to be significantly lower among ITP patients; $2.2 \%$ (3/135) versus controls; 10.1\% (16/158), $\mathrm{p}=0.002 *$. [Table 3]

On comparing the quantitative estimation of antibodies in sera (Table 4), a significantly higher mean level for anti $H$. pylori IgM antibodies was revealed among controls (mean \pm SD $72.14 \mathrm{U} / \mathrm{ml} \pm 22.17$ ), compared to ITP patients (mean \pm SD $56.57 \mathrm{U} / \mathrm{ml}$ \pm 10.85 ) with a $p$ value of 0.013 . However, a higher mean level of $\mathrm{IgG}$ antibodies was detected among ITP patients $(96.27 \mathrm{U} / \mathrm{ml} \pm$ 24.15) when compared to controls (83.73 $\mathrm{U} / \mathrm{ml} \pm 38.37$ ), but without a statistical significance difference between both groups, $\mathrm{p}=0.098$.

On studying the correlation coefficient between the quantitative determination of $\mathrm{H}$ pylori $\operatorname{IgM}$ and $\mathrm{IgG}$ antibodies on one hand and platelet counts of ITP patients on the other, our study proved a statistically significant negative correlation between them i.e. an increase in antibody titre, whether IgM or $\mathrm{IgG}$, is associated with a decrease in platelet counts among ITP patients, with a $p$ value of.0001 for both, as shown in table 5 and figure 1.

The relationship between $H$. pylori and other autoimmune diseases, such as ITP, has motivated us to find out if there is any correlation between anti-H pylori IgM and IgG titres and severity of thrombocytopenia in those patients as a part of studying the pathogenesis of this disease and predicting the cure when administrating anti-H pylori therapy.

The role of $\mathrm{H}$ pylori in the pathogenesis of ITP has been suggested because significant increases in platelet counts were reported after $\mathrm{H}$ pylori eradication. However, the role of $\mathrm{H}$ pylori in the pathogenesis of ITP is still controversial. Several studies have attempted to explain the underlying pathogenic mechanism of $\mathrm{H}$ pylori induced ITP. Most prevailing hypothesis suggests molecular mimicry between one of the $\mathrm{H}$ pylori antigens and platelet glycoproteins is causing production of cross-reacting auto-antibodies (Franchini et al., 2010).

$\mathrm{H}$ pylori are distributed worldwide, though the prevalence strongly varies between developing and developed countries; it is more than 80 and $30 \%$, respectively. An Indian report indicates that almost $80 \%$ of the population is infected with $H$. pylori (Poddar and Yaccha, 2007).

Regarding the association between $\mathrm{H}$ pylori and ITP, Gasbarrini et al., (Gasbarrini et al., 1998) reported that $61 \%$ of 18 ITP cases were infected with $\mathrm{H}$ pylori. Since this report by Gasbarrini et al., an accumulating body of evidence has proposed a patho-physiological link between ITP and chronic $H$. pylori infection. Clinical reports have described a spontaneous resolution of ITP symptoms in about $50 \%$ of chronic ITP patients following empirical treatment of $H$. pylori infection. Emilia et al., (Emilia et al., 2001) then reported that $43 \%$ of 30 ITP patients were $H$. pylori positive. The prevalence of $\mathrm{H}$ pylori infection in healthy population of Italy, where Gasbarrini's and Emilia's studies were held, was about 63\% (Luzza et al., 1998).

In our study, the prevalence of $\mathrm{H}$ pylori seropositivity in ITP patients was $80 \%$, lower than that in the general population $\approx 90 \%$. Moreover, $\mathrm{H}$ pylori IgG sero-positivity was present in $\approx 78 \%$ of ITP patients and in $\approx 80 \%$ of the general population; however, IgM seropositivity was present in $\approx 29 \%$ of ITP patients and in $\approx 54 \%$ of the general population. Thus, there was no significant increase in sero-prevalence of $\mathrm{H}$ pylori among 
ITP patients. Both IgM and IgG titres showed significant negative correlation with the severity of thrombocytopenia in our ITP patients $(r=-.340$ and -.385 , respectively, $\mathrm{p}=$ 0.001 for both).

The finding of low prevalence of ITP among acutely infected individuals and higher prevalence of ITP among those with longstanding infection is acceptable to justify the proposed hypothesis that prolonged exposure to high level of antibodies would contribute to the development of the autoimmune disease. This agrees with researchers disclaiming any direct relation of acute infection with ITP pathogenesis.

Furthermore, this has been confirmed by the significantly negative correlation coefficient revealed between $\mathrm{H}$ pylori $\mathrm{IgG}$ antibody titres and platelet counts, among ITP patients. These data would be of great benefit for clinicians for better understanding of ITP aetiology, thus improving management of such cases and their outcome.

We have proved that $H$. pylori $\operatorname{IgM}$ and $\operatorname{IgG}$ seropositivity is very common and widely spread in Egypt. The prevalence of $H$. pylori seropositivity among adults in this Northern Egyptian community is $89.9 \%$, which is relatively high when compared to its prevalence elsewhere.

This is mostly attributed to the low socioeconomic status, poor human and domestic waste disposal systems and household crowding, which are the main factors that enhance the infection. The high percentages documented in our study can, also, be explained by the reporting of Shukla et al.,(Shukla et al., 2012) that "ELISA, if done alone, may overestimate the presence of active $\mathrm{H}$ pylori infection as antibody titres can remain elevated even after the eradication of H pylori “.
Michel et al., (Michel et al., 2002) and Jargue et al., (Jargue et al., 2001) found no evidence of an association between $\mathrm{H}$ pylori infection and ITP. Kohda et al., (Kohda et al., 2002) found that $\mathrm{H}$ pylori were positive in $62.5 \%$ of 40 ITP patients in Japan, where the prevalence of $\mathrm{H}$ pylori infection ranged between $25-45 \%$. Kurtoglu et al., (Kurtoglu et al., 2004) found $\mathrm{H}$ pylori infection in $65.2 \%$ of healthy Turkish individuals, while its prevalence in Turkish ITP patients was $68.5 \%$.

Kohda et al (Kohda et al., 2002) and Michel et al (Michel et al., 2002) found no significant difference in platelet counts between $\mathrm{H}$ pylori-positive and $\mathrm{H}$ pylori-negative ITP patients. However, in our study, we found a statistically significant decrease in platelet counts among $\mathrm{H}$ pylori sero-positive ITP patients when compared with their seronegative counterparts, $\mathrm{p}<0.05$. Contrary to our findings, the study carried out by Kurtoglu et al., (Kurtoglu et al., 2004) revealed higher platelet counts among $H$. pylori positive group than $\mathrm{H}$ pylori-negative group at the initial presentation, and the difference between the two groups was significant $(\mathrm{p}<0.05)$.

In the review done by Stasi et al., (Stasi et al., 2009), they reported worldwide prevalence of $\mathrm{H}$ pylori in ITP patients from 25 studies. The result from these studies revealed an overall prevalence of $62.3 \%$. However, when matched with age and geographic area, the prevalence rate of $\mathrm{H}$ pylori infection, in most of these studies, were similar to the healthy population. Similarly, Liebman (Liebman, 2007) showed that the prevalence of $\mathrm{H}$ pylori infection in patients with ITP was similar to controls matched for age and geographical location. Also, we found the sero-prevalence of $H$. pylori infection to be $80 \%$ and $89 \%$ in ITP patients and healthy controls respectively. 
Table.1 Distribution of cases and controls according to $H$. pylori sero-positivity

\begin{tabular}{|r|r|l|r|}
\hline & \multicolumn{1}{|c|}{$\begin{array}{c}\text { ITP patients } \\
(\mathbf{n = 1 3 5})\end{array}$} & Controls $(\mathbf{n = 1 5 8})$ & p \\
\hline Total seropositive subjects & $108 / 135(80 \%)$ & $142 / 158(89.9 \%)$ & 0.78 \\
\hline IgM positive subjects $(\mathbf{n})$ & 39 & 86 & $0.004^{*}$ \\
$-\%$ of seropositive subjects & $39 / 108(36.1 \%)$ & $86 / 142(60.6 \%)$ & \\
$-\%$ of total & $39 / 135(28.9 \%)$ & $86 / 158(54.4 \%)$ & 0.54 \\
\hline IgG positive subjects (n) & 105 & 126 & \\
-\% of seropositive subjects & $105 / 108(97.2 \%)$ & $126 / 142(88.7 \%)$ & \\
$-\%$ of total & $105 / 135(77.8 \%)$ & $126 / 158(79.8 \%)$ & \\
\hline
\end{tabular}

ITP: Immune thrombocytopenic purpura p: significant if $<0.05$

Table.2 Comparison between H. pylori sero-positive and sero-negative ITP patients

\begin{tabular}{|r|c|c|c|}
\hline & $\begin{array}{c}\text { H. pylori } \\
\text { seropositive ITP } \\
\text { Patients }\end{array}$ & $\begin{array}{c}\text { H. pylori } \\
\text { seronegative ITP } \\
\text { Patients }\end{array}$ & p \\
\hline $\begin{array}{c}\text { Number of Patients } \\
\text { Platelet count (x10 } / \mathbf{c m m}^{\mathbf{3}} \text { ) } \\
\text { mean (range) }\end{array}$ & $108 / 135(80 \%)$ & $27 / 135(20 \%)$ & \\
\hline
\end{tabular}

H pylori $=$ Helicobacter pylori

ITP: Immune thrombocytopenic purpura

p: significant if $<0.05$

Table.3 Incidence of seropositivity of H. Pylori $\operatorname{IgM}$ and $\mathrm{IgG}$ antibodies in both studied groups

\begin{tabular}{|c|c|c|c|c|}
\hline \multirow{2}{*}{\multicolumn{2}{|c|}{ H pylori Seropositivity (IgM, IgG) }} & \multicolumn{2}{|c|}{ Groups } & \multirow{2}{*}{$\mathbf{X}^{2}$} \\
\hline & & $\begin{array}{c}\text { Controls } \\
(n=158)\end{array}$ & $\begin{array}{l}\text { ITP } \\
(n=135)\end{array}$ & \\
\hline \multirow[t]{2}{*}{ Both -ve } & $\mathrm{n}$ & 16 & 27 & \multirow{2}{*}{0.052} \\
\hline & $\%$ & $10.1 \%$ & $20 \%$ & \\
\hline \multirow[t]{2}{*}{ IgM-ve, IgG +ve } & $\mathrm{n}$ & 56 & 69 & \multirow{2}{*}{0.037} \\
\hline & $\%$ & $35.4 \%$ & $51.1 \%$ & \\
\hline \multirow[t]{2}{*}{ Both +ve } & $\mathrm{n}$ & 70 & 36 & \multirow{2}{*}{$0.003 *$} \\
\hline & $\%$ & $44.3 \%$ & $26.7 \%$ & \\
\hline \multirow[t]{2}{*}{ IgM+ve, IgG-ve } & $\mathrm{n}$ & 16 & 3 & \multirow{2}{*}{$0.002 *$} \\
\hline & $\%$ & $10.1 \%$ & $2.2 \%$ & \\
\hline \multirow[t]{2}{*}{ Total } & $\mathrm{n}$ & 158 & 135 & 293 \\
\hline & $\%$ & $100.0 \%$ & $100.0 \%$ & $100.0 \%$ \\
\hline
\end{tabular}

H pylori = Helicobacter pylori

IgG = Immunoglobulin $\mathrm{G}$

IgM = Immunoglobulin M

ITP = Idiopathic thrombocytopenic purpura

p: significant if $<0.05$ 
Table.4 Comparison between cases and controls regarding H pylori $\operatorname{IgM}$ and $\operatorname{IgG}$ positive titres

\begin{tabular}{|l|l|l|c|}
\hline H pylori Seropositivity & \multicolumn{1}{|c|}{ ITP $(\mathbf{n = 1 3 5})$} & \multicolumn{1}{|c|}{ Controls $(\mathbf{n = 1 5 8})$} & \multicolumn{1}{c|}{, p } \\
\hline IgM $(\mathbf{U} / \mathbf{m l})$ & & & \\
Range & $42-77$ & $40-116.53$ & $\mathrm{t}=3.08, \mathrm{p}=0.013^{*}$ \\
Mean \pm SD & $56.57 \pm 10.85$ & $72.14 \pm 22.17$ & \\
\hline IgG $(\mathbf{U} / \mathbf{m l})$ & & & \\
Range & $21-140$ & $23-131.3$ & $(\mathrm{t}=1.65, \mathrm{p}=0.098)$. \\
Mean \pm SD & $96.27 \pm 24.15$ & $83.73 \pm 38.37$ &
\end{tabular}

H. pylori $=$ Helicobacter pylori

$\operatorname{IgG}=$ Immunoglobulin $\mathrm{G}$

IgM= Immunoglobulin M

p: significant if $<0.05$

Table.5 Correlation between platelet counts and both $H$. pylori

IgM and IgG titres among ITP patients

\begin{tabular}{|l|l|l|}
\hline & \multicolumn{2}{|c|}{ Platelet Counts of ITP Patients } \\
\hline & \multicolumn{1}{|c|}{$\mathbf{~ r}$} & \multicolumn{1}{c|}{ p } \\
\hline H pylori IgM Titre & $-.340^{* *}$ & .0001 \\
\hline H pylori IgG Titre & $-.385^{* *}$ & .0001 \\
\hline
\end{tabular}

H pylori $=$ Helicobacter pylori

IgG = Immunoglobulin $\mathrm{G}$

IgM = Immunoglobulin M

ITP = Idiopathic thrombocytopenic purpura

$\mathbf{r}=$ correlation

p: significant if $<0.05$

Fig.1 Graphical representation of the negative correlation between $H$. pylori $\operatorname{IgG}$ titre and platelet counts among ITP patients

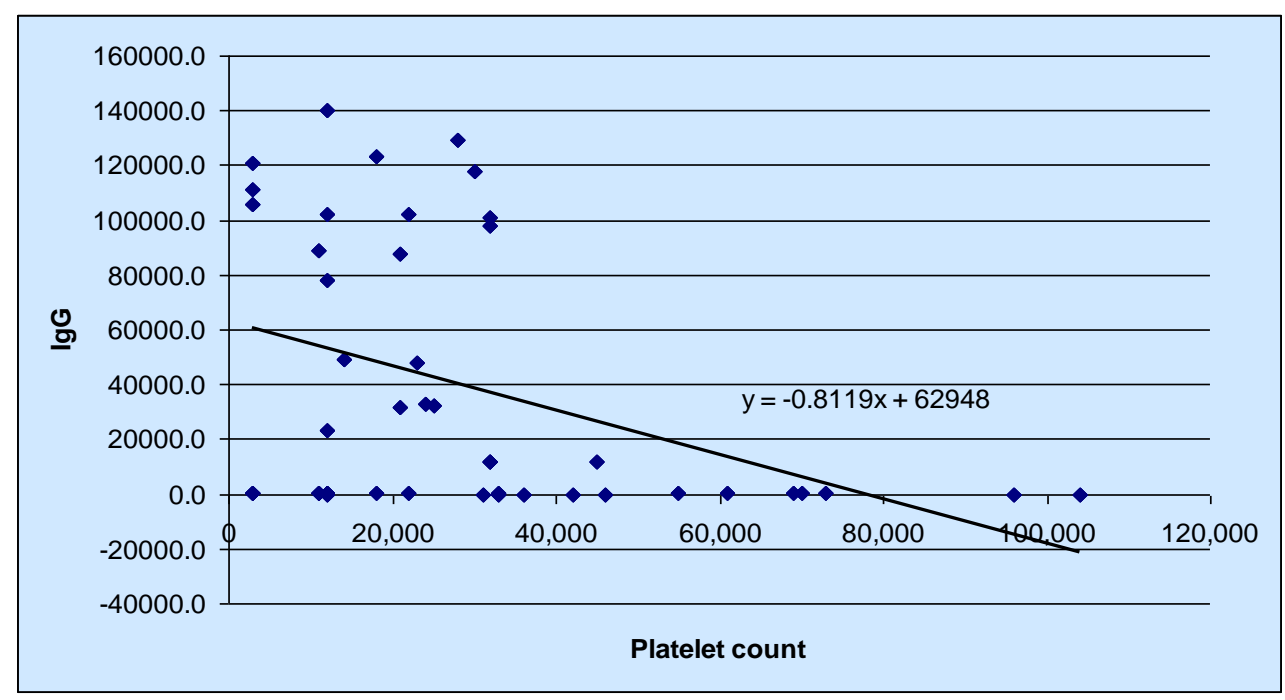


We hypothesized that the severity of ITP might depend on the density of $H$. pylori antibodies i.e. anti-H pylori antibody titre, since bacterial eradication has been proved, by previous studies, to result in platelet count improvement. Serum ELISA for IgM and IgG antibodies against $H$. pylori, done in our study, correlated significantly $(\mathrm{p}<0.001)$ with the severity of thrombocytopenia, which was comparable to a study by Kate et al., (Kate and Ananthakrishnan, 2000).

Studies have also documented that ITP patients in East Asian countries are more likely to express positive antibody titers against $H$. pylori-specific cytotoxic-associated gene A (CagA), a virulence factor that is associated with an increased risk for gastric diseases including carcinoma. While a definitive mechanism by which $H$. pylori may induce thrombocytopenia remains elusive, proposed pathways include molecular mimicry of CagA by host auto antibodies against platelet surface glycoproteins, as well as alterations in the phagocytic activity of monocytes (Frydman et al., 2015).

Results of Abdollahi et al.,(Abdollahi et al., 2015) strongly support the role of $H$. pylori in ITP children, by demonstrating a statistically significant higher prevalence of $H$. pylori stool Ag in ITP cases than in controls. Among the weak points of our study, are the relatively small sample size and antibody quantitation by ELISA, which might overestimate the presence of active $\mathrm{H}$ pylori infection, as antibody titers can remain elevated even after the eradication of $\mathrm{H}$ pylori. However, among the strong points, are the same ethnic origin of the population studied; all Egyptians from Northern Egypt, and the significant negative correlation found between quantitative estimation of $\mathrm{H}$ pylori Ig titers and platelet counts among ITP patients. Further research on the immunological responses to infectious agents, including $\mathrm{H}$ pylori, is still needed. We strongly support the proposal that the detection and eradication of $\mathrm{H}$ pylori could be an effective means for treating ITP. This will, surely, require extensive studies to confirm the suggested causative relationship between bacterial infection and an autoimmune disease state.

In conclusion, the diagnostic work-up for patients with ITP should include tests to detect the presence of $\mathrm{H}$ pylori and to quantitate their antibodies. This conclusion is confirmed by the results of our study. We further recommend that patients with thrombocytopenia, who are also infected with $\mathrm{H}$ pylori, should be treated with traditional triple therapy. $H$. pylori $\mathrm{IgM}$ and $\mathrm{IgG}$ seropositivity is very common and widely spread in Egypt. The prevalence of $H$. pylori seropositivity among adults in this Northern Egyptian community is $89.9 \%$, which is relatively high when compared to its prevalence elsewhere. The incidence of seropositivity for anti-Helicobacter $\operatorname{IgM}$ among controls was found to be significantly higher than among ITP patients. Serum IgM and IgG antibodies against $H$. pylori correlate significantly with the severity of thrombocytopenia. $\mathrm{H}$ pylori infection should be searched in all ITP patients, and we suggest that it should be eradicated in all $\mathrm{H}$ pylori-positive ITP patients.

\section{Acknowledgement}

All authors have fulfilled the following: Substantial contributions to research design, the acquisition, analysis and interpretation of data, Drafting the paper and revising it critically, approval of the submitted and final versions.

\section{References}

Abdollahi, A., Shoar, S., Ghasemi, S., et al., 2015. Is Helicobacter pylori infection a risk factor for idiopathic 
thrombocytopenic purpura in children? Ann. Afr. Med., 14: 177-181.

Amital, H., Govoni, M., Maya, R., et al., 2008. Role of infectious agents in systemic rheumatic diseases. Clin. Exp. Rheumatol., 26: S27-S32.

Binu, V.S., Mayya, S.S., Dhar, M. 2014. Some basic aspects of statistical methods and sample size determination in health science research. Ayu, 35: 119-123.

Bruscky, D.M., da Rocha, L.A., Costa, A.J. 2013. Recurrence of chronic urticaria caused by re-infection by helicobacter pylori. Rev. Paul. Pediatr., 31: 272-275.

Cooke, A., Ferraccioli, G.F., Herrmann, M., et al., 2008. Induction and protection of autoimmune rheumatic diseases, the role of infections. Clin. Exp. Rheumatol., 26: S1-7.

Emilia, G., Longo, G., Luppi, M., et al., 2001. Helicobacter pylori eradication can induce platelet recovery in idiopathic thrombocytopenic purpura. Blood, 97: 812-814.

Franchini, M., Plebani, M., Montagnana, M., et al., 2010. Pathogenesis, laboratory, and clinical characteristics of helicobacter pylori -associated immune thrombocytopenic purpura. Adv. Clin. Chem., 52: 131-144.

Frydman, G.H., Davis, N., Beck, P.L., et al., 2015. Helicobacter pylori eradication in patients with immune thrombocytopenic purpura: A review and the role of biogeography. Helicobacter, 20: 239-251.

Gasbarrini, A., Franceschi, F., Tartaglione, R., et al., 1998. Regression of autoimmune thrombocytopenia after eradication of helicobacter pylori. Lancet, 352: 878.

Hasni, S., Ippolito, A., Illei, G.G. 2011. Helicobacter pylori and autoimmune diseases. Oral Dis., 17: 621-627.

Hasni, S.A. 2012. Role of Helicobacter pylori infection in autoimmune diseases. Curr. Opin. Rheumatol., 24: 429-434.

Jargue, I., Andreu, R., Llopis, I., et al., 2001. Absence of platelet response after eradication of Helicobacter pylori infection in patients with chronic idiopathic thrombocytopenic purpura. $\mathrm{Br}$. J. Haematol., 115: 1002-1003.

Kao, C.Y., Sheu, B.S., Wu, J.J. 2016. Pathogenesis. Biomed. J., 39: 14-23.

Kate, V., Ananthakrishnan, N. 2000. Helicobacter pylori and gastric carcinoma: Evidence for the link. Natl. Med. J. India, 13: 329.

Kohda, K., Kuga, T., Kogawa, K., et al., 2002. Effect of Helicobacter pylori eradication on platelet recovery in Japanese patients with chronic idiopathic thrombocytopenic purpura and secondary autoim- mune thrombocytopenic purpura. $\mathrm{Br} . \quad \mathrm{J}$. Hematol., 118: 584-588.

Kurtoglu, E., Kayacetin, E., Ugur, A. 2004. Helicobacter pylori infection in patients with autoimmune thrombocytopenic purpura. World J. Gastroenterol., 10: 2113-2115.

Liebman, H. 2007. Other immune thrombocytopenias. Semin. Hematol., 44: S24-S34.

Luzza, F., Imeneo, M., Maletta, M., et al., 1998. Suggestion against an oral-oral rout of transmission for Helicobacter pylori infection: a sero-epidemiological study in a rural area. Dig. Dis. Sci., 43: 14881492.

Marshall, B.J., Warren, J.R. 1984. Unidentified curved bacilli in the stomach of patients with gastritis and peptic ulceration. Lancet, 1: 1311-1315.

Michel, M., Khellaf, M., Desforges, L., et al., 2002. Autoimmune thrombocytopenic purpura and Helicobacter pylori infection. Arch. Intern. Med., 162: 1033-1036.

Poddar, U., Yaccha, S.K. 2007. Helicobacter pylori in children: An Indian perspective. Indian Pediatr., 44: 761-770.

Rizzo, R., Bortolotti, D., Bolzani, S., Fainardi, E. 2014. HLA-G molecules in autoimmune diseases and infections. Front. Immunol., 18: 592.

Sherman, P.M., Lin F.Y. 2005. Extra-digestive manifestation of helicobacter pylori infection in children and adolescents. Can. J. Gastroenterol., 19: 421-424. 
Shmuely, H., Domniz, N., Yahav, J. 2016. Nonpharmacological treatment of helicobacter pylori. World J. Gastrointest. Pharmacol. Ther., 7: 171-178.

Shukla, S., Pujani, M., Agarwal, A., et al., 2012. Correlation of serology with morphological changes in gastric biopsy in Helicobacter Pylori infection and evaluation of immuno-histochemistry for $H$. pylori identification. Saudi $J$. Gastroenterol., 18: 369-374.

Stasi, R., Willis, F., Shannon, M.S., et al., 2009. Infectious causes of chronic immune thrombocytopenia. Hematol. Oncol. Clin. North Am., 23: 1275-1297.

Wong, F., Rayner-Hartley, E., Byrne, M.F. 2014. Extra-intestinal manifestations of helicobacter pylori: a concise review. World J. Gastroenterol., 20: 1195011961.

Zhong, Y., Anderl, F., Kruse, T., et al., 2016. Helicobacter pylori HP0231 influences bacterial virulence and is essential for gastric colonization. PLoS One, 11: e0154643.

\section{How to cite this article:}

Nesren F. Hanafi and Doreen N. Younan. 2017. Prevalence of Helicobacter pylori Antibodies in Egyptians with Idiopathic Thrombocytopenic purpura and In the General Egyptian Population: A Comparative Study. Int.J.Curr.Microbiol.App.Sci. 6(5): 2482-2492. doi: https://doi.org/10.20546/ijcmas.2017.605.278 